\title{
Prevalencia de condilomas ano-genitales en pacientes con infección por VIH en un centro para infecciones de transmisión sexual en León, Guanajuato, México
}

\author{
Prevalence of anogenital condylomas in patients with HIV infection in a center \\ for sexually transmitted infections in Leon, Guanajuato, Mexico
} Carlos Daniel Sánchez-Cárdenas', Gregorio López-Salinas², Silvia Ramírez-Dóvala³, Eder Rodrigo Juárez-Durán",
Pedro Antonio Salceda-Muñoz, Juan Luis Mosqueda-Gómez y y Gabriela Moreno-Coutiño

\author{
'Universidad de Guanajuato, Guanajuato, México. \\ ${ }^{2}$ CAPASITS León, Guanajuato, México. \\ ${ }^{3}$ Hospital General de León. \\ ${ }^{4}$ Hospital General "Dr. Manuel Gea González". \\ 5Universidad de Guanajuato, México. \\ ${ }^{6}$ Hospital Regional de Alta Especialidad del Bajío. \\ ${ }^{7}$ Hospital General "Dr. Manuel Gea González"
}

Los autores declaramos no tener ningún conflicto de interés.

Financiamiento: Ninguno.

Recibido: 11 de noviembre de 2019 (segunda versión: 2 de noviembre de 2020) / Aceptado: 12 de enero de 2021

\section{Resumen}

Introducción: Los condilomas son la infección de transmisión sexual más frecuente en el mundo y tiene estrecha relación con la infección por VIH. La topografía y la serología son variables, y algunos presentan mayor riesgo de malignización. Objetivo: Describir la prevalencia, características clínicas, topográficas y factores asociados a la presencia de condilomas ano-genitales en pacientes con infección por VIH/SIDA en Guanajuato. Material y Métodos: Estudio descriptivo y transversal realizado entre junio y diciembre de 2014. Se incluyeron pacientes adultos con infección por VIH/SIDA, de ambos sexos, del CAPASITS León, México. Se aplicó un cuestionario que incluyó: datos de identificación, y factores de riesgo relacionados a los condilomas ano-genitales, y se les revisó dicha región corporal en busca de condilomas. Se realizó estadística descriptiva de acuerdo al tipo y distribución de las variables y se compararon las medianas de linfocitos T CD4 (LTCD4+) de los pacientes, con y sin condilomas, con la prueba U- Mann-Whitney. Resultados: Se incluyeron 213 pacientes con infección por VIH/SIDA, 181 (85\%) hombres. La prevalencia de condilomas ano-genitales fue de 30\% (IC95\%:23-36\%), con mayor frecuencia en la región anal, con $21 \%$ de los casos. Encontramos una

\begin{abstract}
Background: Condylomas are the most frequent sexually transmitted infection worldwide, and thus, have a close relation to HIV infection. The site and serology vary, and some pose higher risk of malignancy. Aim: To describe the prevalence, characteristics and associated factors to the presence of anogenital condylomas in patients with HIV/AIDS. Methods: Descriptive cross-sectional study conducted in the period from June to December 2014. Adult HIV/AIDS positive patients from "CAPASITS" in Leon, Mexico, from both sexes were included. A questionnaire was performed that included: identification data, and risk factors related to anogenital condylomas. Anogenital area was examined for condylomas. Descriptive statistics were carried out according to the type and distribution of the variables, and the CD4 lymphocyte medians of patients with and without condylomas were compared using U- Mann-Whitney test. Results: A total of $213 \mathrm{HIV} /$ AIDS patients were included; $181(85 \%)$ were male. The prevalence of anogenital condylomas was 30\% (IC95\%: 23-36\%), predominating in the anal region, observed in $21 \%$ of the cases. A significant difference was found between median CD4 cell count of patients with and without condylomas (425 vs $510 \mathrm{CD} 4 / \mathrm{mL}, \mathrm{p}=0.034)$. Conclusion: A
\end{abstract}


diferencia significativa entre las medianas en el recuento de linfocitos LTCD4+ en los pacientes, con y sin condilomas, (425 vs 510 LTCD4+/ $\mathrm{mL}, \mathrm{p}=0,034)$. Conclusiones: Se obtuvo una prevalencia alta de pacientes con condilomas ano-genitales. La presencia de condilomas fue mayor en pacientes con menor recuento de linfocitos LTCD4+. Considerando la elevada prevalencia, se recomienda la aplicación de la vacuna en este grupo de pacientes.

Palabras clave: VIH; SIDA; VPH; condiloma; prevalencia.

\section{Introducción}

E 1 virus papiloma humano (VPH) causa la infección de transmisión sexual (ITS) más frecuente en el mundo y tiene especial asociación con la infección por VIH, con la cual se altera la historia natural de la enfermedad y aumenta su prevalencia. Se le conocen muchos genotipos que comúnmente se dividen en virus de bajo riesgo para malignidad (genotipos 6 y 11), que causan verrugas, y los de alto grado (genotipos 16 y 18) que ocasionan displasias, neoplasias intra-epiteliales y cáncer. Sin embargo, hay muchos otros genotipos que han sido identificados en la zona ano-genital y cada vez hay más evidencia de que la división entre alto y bajo riesgo es poco precisa $^{1,2}$.

Los condilomas pueden ser milimétricos o medir varios centímetros $\mathrm{y}$ en ocasiones, por la localización, pueden pasar desapercibidas, pero cuando se tiene el diagnóstico, suelen generar estrés psicológico e incomodidad física.

Afecta a ambos sexos, con predilección en hombres que tienen sexo con hombres (HSH), con incidencias que van de 2 a $84 \%$. Veinte a $30 \%$ serán infecciones persistentes y aquellas de alto riesgo son responsables de $80 \%$ del cáncer anal ${ }^{3}$.

Es frecuente que pacientes infectados por VIH presenten infección por VPH, especialmente por los genotipos de alto riesgo para malignidad, como es el 16, por lo que se les considera un grupo de alto riesgo para cáncer anal ${ }^{4,5}$. Además, en estos pacientes, la infección por VPH se asocia a bajas recuentos de linfocitos CD4 LTCD4+, y aún no se ha podido determinar si los anti-retrovirales reducen la incidencia, pero sí se ha visto que no reducen la incidencia de cáncer en los que ya tienen neoplasia intra-epitelial ${ }^{4}$.

En México, no hay suficiente información acerca de las infecciones ano-genitales de los pacientes con infección por VIH/SIDA, específicamente condilomas ano-genitales. El objetivo de este estudio es conocer la prevalencia, distribución y factores predisponentes de los condilomas ano-genitales de pacientes con infección por VIH/SIDA en un centro de atención para ITS en León, Guanajuato, México.

\section{Métodos}

Se llevó a cabo un estudio descriptivo y transversal en pacientes con infección por VIH, durante el período de junio a diciembre de 2014, en el Centro Ambulatorio para la Prevención y Atención en SIDA e high prevalence of patients with anogenital condylomas was obtained. The presence of condylomas was higher in patients with lower CD4 count. Considering the high prevalence, the application of the vaccine is recommended in this patient group.

Keywords: HIV; AIDS; HPV; condyloma; prevalence

Infecciones de Transmisión Sexual (CAPASITS) de la ciudad de León, Guanajuato, México.

El estudio fue aprobado por el Comité de Ética e Investigación del Hospital General de León, Guanajuato.

Se invitó a participar a todos los pacientes mayores de 18 años, de ambos sexos, que acudieron a seguimiento de su enfermedad (VIH/ SIDA) en el CAPASITS León. Firmaron un consentimiento informado y posteriormente se les hicieron preguntas para recabar información socio-demográfica y acerca de factores de riesgo para adquirir condilomas ano-genitales. A todos los participantes se les realizó una inspección de la región ano-genital por un especialista en Dermatología en búsqueda de lesiones compatibles con condilomas. Los datos obtenidos se virtieron en una hoja de captura para su posterior análisis estadístico con el programa IBM SPSS Statistics v23.

Los datos se presentan utilizando estadística descriptiva (promedio, media con desviación estándar o medianas y rangos). La comparación de los grupos se realizó con $\chi^{2}$, t de Student y U Mann-Whitney según correspondiera.

\section{Resultados}

Se incluyeron 213 pacientes, de los cuales 181 (85\%) fueron del sexo masculino. La media de edad de la población fue de 39,4 años ( $\pm 11,4 \mathrm{DS})$. No hubo diferencia en el promedio de edad entre hombres y mujeres $(39,2$ vs $40,8, p=0,472)$.

Se encontraron condilomas ano-genitales en 63 de los 213 pacientes, lo que corresponde a una prevalencia de 29,5\% (IC95\%: 24-36\%). No se encontró una diferencia significativa en la prevalencia de condilomas ano-genitales al comparar hombres vs mujeres ( 30,9 vs $21,8 \% \mathrm{p}=0,3)$. (Figura 1).

El tipo de relación sexual anal-receptivo fue más frecuente en hombres que en mujeres $(65,7$ vs $6,2 \%, p<0,001)$. No se encontró diferencia significativa en la prevalencia de condilomas anales al comparar a las personas que referían actividad sexual anal receptiva y las que no $(25,6$ vs $15,2 \%, p=0,065)$.

De los pacientes con condilomas, 26 (41,3\%) tenían el antecedente de tabaquismo. No se encontró una diferencia significativa en la prevalencia de condilomas entre los pacientes fumadores comparado con los no fumadores ( 32 vs $28 \% \mathrm{p}=0,528$ ).

En 18 pacientes (28\%) las lesiones se localizaron en el área genital y anal y en $45(71,4 \%)$ sólo en la región anal/perianal (Figura 1). 


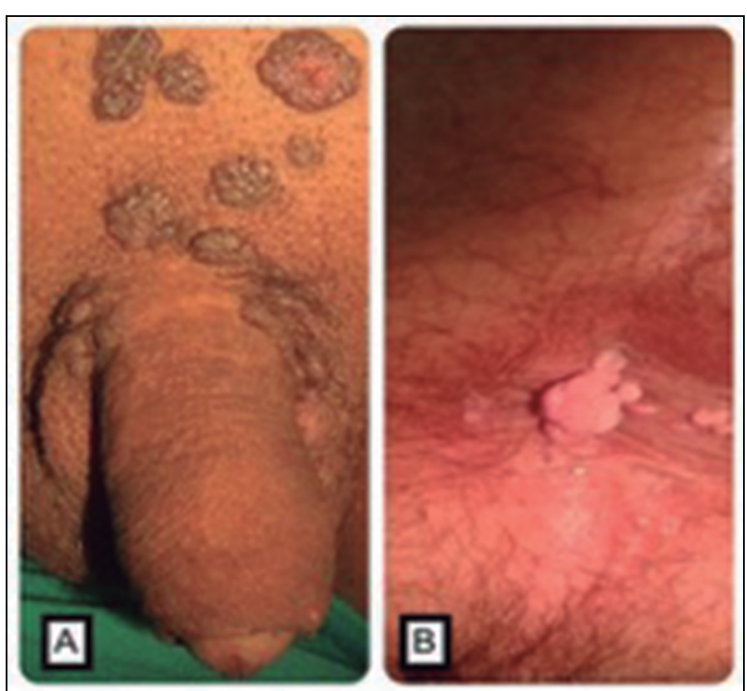

Figura 1.

Al analizar los datos por sexo, de los 56 hombres con condilomas, $39(70 \%)$ los presentaron en la región anal y $17(30 \%)$ en la región genital-anal; de las 7 mujeres con condilomas, $6(86 \%)$ los presentaron en la región anal y $1(14 \%)$ en la región genital.

La mediana de LTCD4+ fue significativamente menor en los pacientes con condilomas al compararlos con los que no tuvieron estas lesiones (425 vs $510 \mathrm{LTCD} 4+/ \mathrm{mL}$, $\mathrm{p}=0,034)$.

De los pacientes que presentaban condilomas, sólo $21 \%$ sabía que los tenían, ya fuera porque los habían identificado por sí mismos o porque habían sido diagnosticados previamente por su médico.

\section{Discusión}

La infección por VPH tiene algunas particularidades: es la ITS de mayor incidencia mundial y, muy frecuentemente, se diagnostica en pacientes infectados por VIH, además de que, junto a la infección por virus de hepatitis $\mathrm{B}$, es una ITS que cuenta con una vacuna como profilaxis. Los condilomas son la lesión ano-genital más frecuente y benigna de la infección por el VPH, con una frecuencia de 40 a $60 \%$ en pacientes con VIH/SIDA, un período de incubación corto, y rápida progresión ${ }^{6-8}$. En nuestro estudio, la prevalencia de los condilomas ano-genitales fue de $29,5 \%$, hallazgo similar a los resultados de estudios realizados en otros países como el publicado por De Camargo y cols., en Brasil (31\%)

En los pacientes con infección por VIH/SIDA, estas lesiones tienden a ser de mayor tamaño, persistentes y con un riesgo más alto de co-infectarse por serotipos oncogénicos que pueden derivar en neoplasia intra-epitelial de alto grado, lo que se ha visto en el caso de cáncer cervical en mujeres con infección por $\mathrm{VIH}^{10-12}$ como observamos en nuestros casos.

Lo anterior ha sido documentado por Anderson y otros autores, quienes describen mayor prevalencia, donde se ve mayor prevalencia de progresión de condilomas a neoplasias malignas en pacientes inmunosuprimidos por el $\mathrm{VIH}^{13-15}$

Es importante resaltar que la prevalencia de condilomas ano-genitales en nuestro trabajo no difirió entre hombres y mujeres, en concordancia con el estudio de Dhumele y cols., realizado en la India ${ }^{16}$.

Dentro de los factores de riesgo para infección por VPH, las prácticas sexuales son muy relevantes, principalmente la anal receptiva, aunque se sabe que también hay casos de auto-inoculación dígito-anal o con fómites. También se han identificado el uso irregular de la terapia anti-retroviral y la co infección con herpesvirus ${ }^{3,17}$.

No encontramos diferencias entre lesiones anales de pacientes con relaciones sexuales anales receptivas y no receptivas. En cuanto a la edad, hay reportados dos picos de mayor incidencia, entre los 20-29 años y después a los 40 , donde se encontraba la mayoría de nuestros pacientes.

Otros factores de riesgo son el número de parejas sexuales en el último año, la infección por VIH y HSH. ${ }^{1}$ También lo son el comienzo de relaciones sexuales a edades tempranas, hombres no circuncidados, tabaquismo $\mathrm{y}$, muy importante, el desconocimiento de padecer la enfermedad, lo cual no permite tener medidas preventivas como lo fue en este estudio, donde sólo $21 \%$ conocía su diagnóstico ${ }^{6}$.

Low y cols., describieron que el consumo de tabaco incrementa tres veces el riesgo de adquirir condilomas ano-genitales ${ }^{18}$. En nuestro estudio, no encontramos una diferencia significativa entre el consumo de tabaco y los condilomas ano-genitales.

Con respecto a la distribución, Dhumale y Sarzo, encontraron que en pacientes con infección por VIH/SIDA es más frecuente el desarrollo de condilomas anales que los genitales ${ }^{8,16,19}$. En nuestro estudio, se obtuvo la misma distribución de las lesiones, tanto en hombres como en mujeres.

El número bajo de LTCD4+ $(\leq 200$ céls $/ \mu 1)$ es un factor predictor para la infección de genotipos de VPH de alto riesgo y también se ha asociado a un incremento en el riesgo de condilomas ano-genitales ${ }^{20}$. Low y cols., no encontraron evidencia acerca de la relación entre el recuento bajo de LTCD4+ y la aparición de condilomas ano-genitales ${ }^{18}$. En nuestro estudio, encontramos una diferencia significativa entre el recuento bajo (menos de 425 céls/ $\mu$ l) de LTCD4+ y la presencia de condilomas.

Woodhall y cols. encontraron un deterioro significativo de la calidad de vida de los pacientes que padecen de condilomas ano-genitales ${ }^{20}$, además de los altos costos 
económicos y sociales que conllevan su tratamiento, aún de las lesiones benignas, por lo que es altamente recomendable la aplicación de la vacuna ${ }^{21}$. En este momento, en México a pesar de que se recomienda la aplicación de la vacuna en pacientes con infección por VIH, no está considerada dentro del esquema de inmunizaciones. Sólo se aplica a niñas de 11 años y en algunos estados también incluyen a los varones de la misma edad.

\section{Conclusiones}

Los pacientes infectados con VIH están en mayor riesgo de padecer infección por VPH la que puede ser recurrente o persistente y, en algunos casos, evolucionar hacia neoplasias intra-epiteliales o invasoras, con las consecuentes repercusiones en la salud, la calidad de vida y mayores costos en la atención médica. Por ese motivo, la educación sexual en la edad escolar resulta muy importante, así como advertir de los factores de riesgo. En el caso particular de los pacientes con infección por VIH, se recomiendan revisiones periódicas para una pronta detección de estas lesiones, además de recomendar la aplicación de la vacuna cuadrivalente, que afortunadamente ya está en el esquema de vacunación de muchos países latinoamericanos, de los cuales Panamá fue pionero y esperemos que México se una a este grupo en un futuro cercano.

\section{Referencias bibliográficas}

1.- Dareng E O, Adebamowo S N, Famooto A, Olawande O, Odutola M K, Olaniyan Y, et al. Prevalence and incidence of genital warts and cervical human papillomavirus infections in Nigerian women. BMC Infect Dis 2019; 19: 27. doi: 10.1186/s12879-018-3582-y.

2.- Werner R N, Westfechtel L, Dressler C, Nast A. Anogenital warts and other HPV-associated anogenital lesions in the HIV-positive patient: a systematic review and meta-analysis of the efficacy and safety of interventions assessed in controlled clinical trials. Sex Transm Infect 2017; 0: 1-8 doi:10.1136/ sextrans-2016-053035.

3.- Chikandiwa A, Chimoyi L, Pisa P T, Chersich M F, Muller E E, Michelow P, et al. Prevalence of anogenital HPV infection, related disease and risk factors among HIV-infected men in inner-city Johannesburg, South Africa: baseline findings from a cohort study. BMC Public Health 2017; 17 (Suppl 3): 425. https:// doi.org/10.1186/s12889-017-4354-0.

4.- Chikandiwa A, Kelly H, Sawadogo B, Ngou J, Pisa P T, Gibson L, et al, HARP Study Group. Prevalence, incidence and correlates of low risk HPV infection and anogenital warts in a cohort of women living with HIV in Burkina Faso and South Africa. PLoS ONE 13 (5): e0196018 https://doi.org/10.1371/journal.pone.0196018.

5.- Furukawa S, Uota S, Yamana T, Sahara R, Lihara K, Yokomaku Y, et al. Distribution of human papillomavirus genotype in anal condiloma acuminatum among Japanese men: the higher prevalence of high risk human papillomavirus in men who have sex with men with HIV infection. AIDS Res Hum Retroviruses 2018; 34: 375-81. doi: 10.1089/ AID.2017.0197.

6.- Nowak R, Gravitt P, He X, Ketende S, Dauda $\mathrm{W}, \mathrm{Omuh} \mathrm{H}$, et al. Prevalence of anal high- risk human papillomavirus infections among HIV-positive and HIV-negative men who have sex with men in Nigeria. Sex Transm Dis. 2016; 43(4): 243-8. doi: 10.1097/ OLQ.0000000000000431.

7.- Tamer E, Cakmak SK, Ilhan MN, Artuz F. Demographic characteristics and risk factors in Turkish patients with anogenital warts. J Infect Public Health. 2016; 9(5): 661-6. doi: 10.1016/j.jiph.2015.12.009.

8.- Sarzo G, Del Mistro A, Mistro A, Finco C, Frayle-Salamanca H, Marino F, et al. Extensive anal condylomatosis: prognosis in relation to viral and host factors. Colorectal Dis [Internet]. 2010; 12( 7): e128-34. https://doi.org/10.1111/ j.1463-1318.2009.01902.x.

9.- de Camargo C C, Tasca K I, Mendes M B, Miot H A, de Souza L D R. Prevalence of anogenital warts in men with HIV/AIDS and associated factors. Open AIDS J. 2014; 8: 2530. doi: 10.2174/1874613601408010025.

10.- Adler D, Wallace M, Bennie T. Cumulative impact of HIV and multiple concurrent Human papillomavirus infections on the risk of cervical dysplasia. Adv Virol. 2016; 1-5. doi: 10.1155/2016/7310894.

11.- Munoz N, Bosch F, de Sanjose S, Herrero R, Castellsagué X, Shah KV, et al. Epidemiologic classification of human papillomavirus types associated with cervical cancer. N Engl J Med. 2003; 348: 518-27. doi: 10.1056/NEJMoa021641.

12.- Ryan D, Compton C, Mayer R. Carcinoma of the anal canal. N Engl J Med. 2000; 342: $792-$ 800. doi: 10.1056/NEJM200003163421107.

13.- Byars R, Poole G, Barber W. Anal carcinoma arising from condyloma acuminata. Am Surg. 2001; 67: 469-72. PMID: 11379652

14.- Anderson C, Boller A, Richardson C, Balcos E, Zera R. Anal condyloma: a comparison between HIV positive and negative patients. Am Surg. 2004; 70: 1014-8. PMID: 15586518
15.- Chaturvedi A, Madeleine M, Biggar R, Engels E. Risk of human papillomavirus-associated cancers among persons with AIDS. J Natl Cancer Inst. 2009; 101: 1120-30. doi: 10.1093/ jnci/djp205.

16.- Dhumale S, Sharma S, Gulbake A. Ano-genital warts and HIV status-a clinical study. J Clin Diagnostic Res. 2017;11(1): WC01-WC04. doi: 10.7860/ JCDR/2017/24610.9171.

17.- Galea J T, León S R, Peinado J, Calvo G, Zamora J, Sánchez H, et al. HPV knowledge, burden and genital wart location among heterosexually identified versus homosexually identified men who have sex with men in Lima, Peru: cross sectional results from a cohort study. BMJ Open 2017; 7:e017338 doi:10.1136/ bmjopen-2017-017338.

18.- Low AJ, Clayton T, Konate I, Nagot N, Ouedraogo A, Huet C, et al. Genital warts and infection with human immunodeficiency virus in high-risk women in Burkina Faso: A longitudinal study. BMC Infect Dis 2011; 11 (1): 20. doi: 10.1186/1471-2334-11-20.

19.- Chu Q, Vezeridis M, Libbey N, Wanebo H. Giant condyloma acuminatum (BuschkeLowenstein tumor) of the anorectal and perianal regions. Analysis of 42 cases. Dis Colon Rectum. 1994; 37: 950-7. doi: 10.1007/ BF02052606.

20.- Silverberg M, Ahdieh L, Munoz A, Anastos K, Burk R, Cu-Uvin S, et al. The impact of HIV infection and immunodeficiency on human papillomavirus type 6 or 11 infection and on genital warts. Sex Transm Dis. 2002; 29 : 427-35. doi: 10.1097/00007435-20020800000001 .

21.- Mohammed H, Blomquist P, Ogaz D, Duffell S, Furegato M, Checchi M, et al. 100 years of STIs in the UK: a review of national surveillance data. Sex Transm Infect 2018; 0: 1-6. doi:10.1136/sextrans-2017-053273. 